\title{
Impact De La Divagation Des Bovins Sur Les Cultures Dans La Ville De Korhogo
}

\author{
Gatien Konan Gboko Brou, \\ Laboratoire de Biologie, de Production et de Santé Animale, \\ Institut de Gestion Agropastorale (IGA), \\ Université Peleforo Gon Coulibaly (UPGC) de Korhogo

\section{Guy Kouassi Brou,} \\ Laboratoire de Biologie végétale, UFR des Sciences Biologiques, \\ Université Peleforo Gon Coulibaly (UPGC) de Korhogo
}

\section{Seguena Fofana,}

Laboratoire de Biologie Végétale, Production Végétale et Protection des

Végétaux, Institut de Gestion Agropastorale (IGA),

Université Peleforo Gon Coulibaly (UPGC) De Korhogo

\section{René Yadé Soro,}

Laboratoire de Biochimie et de Microbiologie,

Institut de Gestion Agropastorale (IGA),

Université Peleforo Gon Coulibaly (UPGC) de Korhogo

Doi:10.19044/esj.2020.v16n15p223 URL:http://dx.doi.org/10.19044/esj.2020.v16n15p223

\section{Résumé}

Une étude a été menée dans la ville de Korhogo en vue de déterminer l'impact de la divagation des bovins sur les cultures. Pour ce faire, une enquête a été menée auprès de 90 cultivateurs et 50 éleveurs. Les résultats ont montré que les éleveurs et les cultivateurs étaient en majorité des hommes contre une minorité de femmes. Les principales races de bovins élevées ont été par ordre d'importance les métis, les zébus, les baoulés et les N'damas. Le mode d'élevage prédominant a été la divagation où les bovins ont été principalement nourris avec le fourrage naturel et abreuvés dans les cours d'eau. La divagation des bovins dans les champs a entrainé beaucoup de dégâts au niveau des différentes cultures. Les cultures annuelles ont été les plus endommagées suivies des cultures maraîchères. Les cultures pérennes ont été moins attaquées. Les causes des dégâts ont été identifiées aussi bien chez les éleveurs que chez les cultivateurs. Les conséquences ont été observées aussi bien chez les bovins que les cultures. En définitive, une sensibilisation serait nécessaire dans le monde agricole en vue de limiter les dégâts de la divagation des bovins. 
Mots clés : Bovins, Divagation, Dégâts, Cultures, Korhogo

\title{
Impact of Cattle Ramblings on Crops in the City of Korhogo
}

\author{
Gatien Konan Gboko Brou, \\ Laboratoire de Biologie, de Production et de Santé Animale, \\ Institut de Gestion Agropastorale (IGA), \\ Université Peleforo Gon Coulibaly (UPGC) de Korhogo \\ Guy Kouassi Brou, \\ Laboratoire de Biologie végétale, UFR des Sciences Biologiques, \\ Université Peleforo Gon Coulibaly (UPGC) de Korhogo \\ Seguena Fofana, \\ Laboratoire de Biologie Végétale, Production Végétale et Protection des \\ Végétaux, Institut de Gestion Agropastorale (IGA), \\ Université Peleforo Gon Coulibaly (UPGC) De Korhogo \\ René Yadé Soro, \\ Laboratoire de Biochimie et de Microbiologie, \\ Institut de Gestion Agropastorale (IGA), \\ Université Peleforo Gon Coulibaly (UPGC) de Korhogo
}

\begin{abstract}
A study was carried out in the city of Korhogo to determine the impact of cattle rambling on crops. To do this, a survey was conducted among 90 growers and 50 breeders. The results showed that the majority of breeders and cultivators were men against a minority of women. The main breeds of cattle raised were, in order of importance, the mestizos, the zebus, the baoulés and the N'damas. The predominant breeding method was straying where cattle were mainly fed with natural fodder and watered in the rivers. The rambling of cattle in the fields has caused a lot of damage in different crops. Annual crops were the most damaged, followed by vegetable crops. Perennial crops have been less attacked. The causes of the damage have been identified for both breeders and cultivators. The consequences have been observed in both cattle and crops. Ultimately, awareness would be necessary in the agricultural world in order to limit the damage caused by the ramblings of cattle.
\end{abstract}

Keywords: Cattle, Ramblings, Damage, Crops, Korhogo 


\section{Introduction}

La base de l'économie nationale est constituée par l'agriculture qui emploie les deux tiers (2/3) de la population active et contribue au Produit Intérieur Brut (PIB) total pour 34\% et aux recettes d'exportation pour $66 \%$. L'élevage reste encore une activité économique en développement avec une contribution d'environ 4,5\% au PIB agricole et $2 \%$ au PIB total. Les productions animales permettent non seulement d'améliorer le statut nutritionnel des populations, mais aussi et surtout, procurent des revenus à plusieurs franges des populations à travers les échanges commerciaux (Coulibaly, 2013). La Côte d'Ivoire connait une situation précaire au niveau de la couverture de ses besoins en protéine bovine (Yeo, 2017) alors que la région Nord du pays dispose de nombreux atouts favorables à l'élevage de bovins (Guen, 2008). Les troupeaux transhumants évoluent dans la région frontalière du Mali et du Burkina Faso. Ce cheptel numériquement important est souvent fractionné en de nombreux troupeaux restreints, qui transhument sur l'ensemble de la zone septentrionale et, dans une moindre mesure, méridionale sur le principe du semi-nomadisme qui consiste pour les bergers ou pasteurs peulhs qui en ont la charge, à résider dans un campement à partir duquel s'effectuent des allers-retours en fonction des pâturages disponibles. Lorsque tout se passe bien entre éleveurs et cultivateurs, les éleveurs ont en quelque sorte un droit de vaine pâture ou de pacage sur les terres en jachère ou en friche (Lhoste et Milleville, 2004). Le phénomène de la divagation des bovins donne entière liberté aux animaux, même si les récoltes ne sont pas entièrement terminées. Cette pratique qui provoque naturellement des dégâts aux cultures, est source de conflits, de tensions entre les éleveurs et les cultivateurs. L'objectif général de ce travail est d'identifier les causes et les conséquences de la divagation des bovins sur les cultures dans la commune de Korhogo.

\section{Materiel Et Methodes Situation géographique}

Korhogo est le chef-lieu du district des savanes et de la région du Poro. Korhogo est une ville située au nord de la Côte d'Ivoire à 648 km d'Abidjan, la capitale économique. C'est à la fois une commune, un chef-lieu de souspréfecture et de département. Le département de Korhogo fait frontière avec les départements suivants : M'Bengué et Tengrela au Nord, Dikodougou et Mankono au sud, Sinematiali à l'Est et Boundiali à l'Ouest (Anonyme, 2014).

\section{Technique d'échantillonnage}

Cette étude a été menée auprès de 140 paysans dont 50 éleveurs, 30 cultivateurs de cultures pérennes, 30 cultivateurs de cultures annuelles et 30 cultivateurs de cultures maraîchères tous résidant dans la commune de 
Korhogo précisément dans quatre quartiers que sont SODEPRA, Marcory, Natiokobadara et Logokaha et dans trois villages à savoir Waraniené, Gnelelekaha et Lenivogo (Figure 1). Un questionnaire a été conçu pour mieux effectuer l'enquête auprès des agriculteurs et des éleveurs. Ce questionnaire est constitué de questions fermées et de questions ouvertes.

\section{Collecte des données}

La collecte des données a été faite par voie d'enquête en deux phases dont une pré-enquête et une enquête proprement dite. La pré-enquête a consisté à se rendre sur le terrain en vue d'identifier les éventuels éleveurs de bovins, les agriculteurs et d'évaluer la pertinence des questionnaires. Au terme de cette phase, des aménagements ont été apportés aux questionnaires. L'enquête proprement dite a consisté à rechercher et à identifier l'itinéraire de divagation des bovins. Les langues utilisées au cours de cette enquête étaient le malinké, le senoufo et le français.

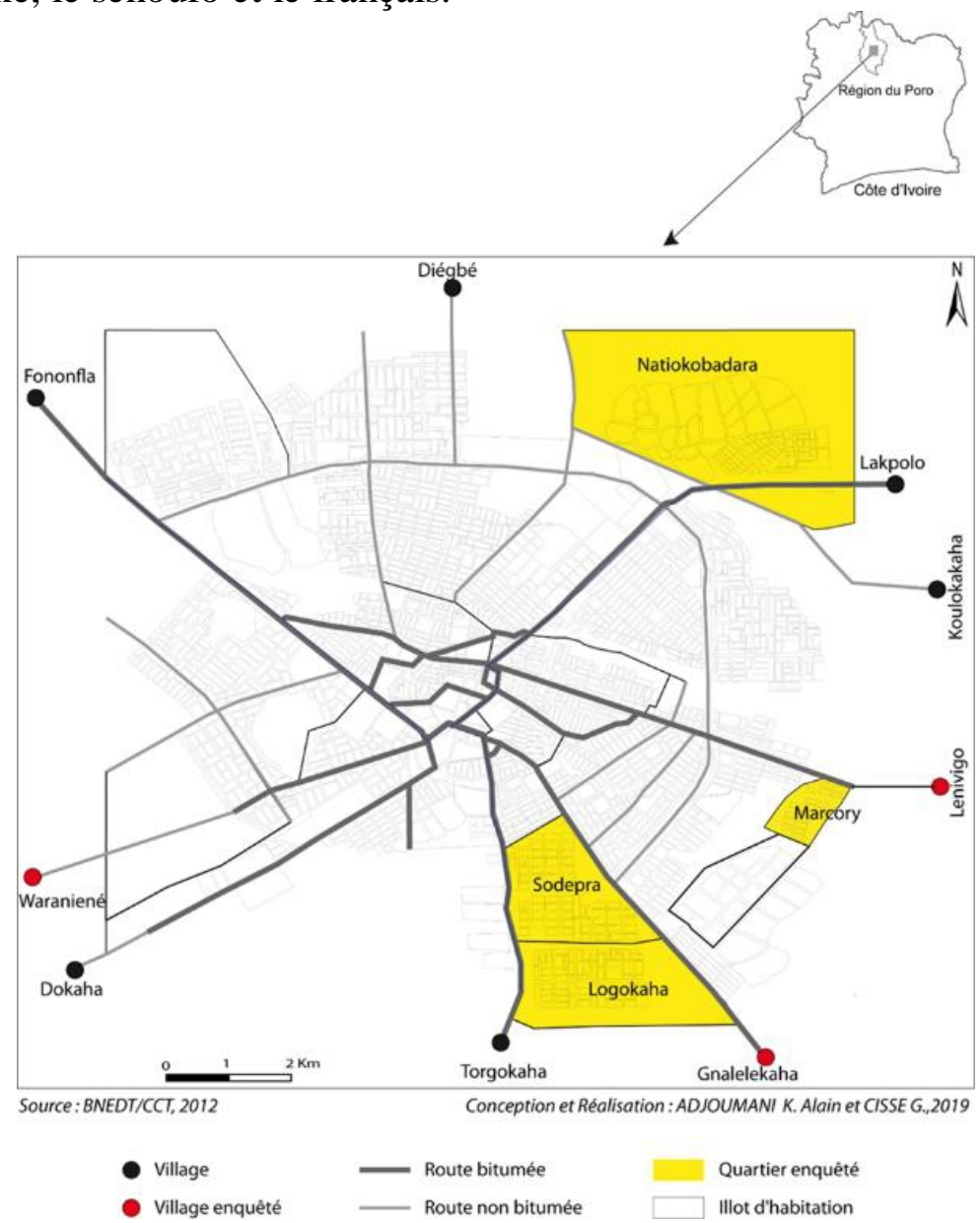

Figure 1: Carte de la ville de Korhogo (Anonyme, 2014) 


\section{Traitement des données}

Après un dépouillement manuel des fiches d'enquête, les résultats ont été traités à l'aide du logiciel Microsoft Excel utilisé pour l'analyse descriptive des données et la production des graphiques.

\section{Resultats}

\section{Profil sociodémographique des cultivateurs et des éleveurs \\ Genre et âge}

Les tableaux 1 et 2 présentent le genre et l'âge des éleveurs et cultivateurs. La majorité de ces acteurs sont des hommes ( $94 \%$ des éleveurs et $69 \%$ des cultivateurs) contre $6 \%$ et $31 \%$ respectivement pour les éleveurs et les cultivateurs qui sont des femmes.

Les acteurs les plus nombreux ont l'âge compris entre 41 et 50 ans ( $48 \%$ d'éleveurs et $40 \%$ de cultivateurs). Les moins nombreux sont ceux dont l'âge est compris entre 20 et 40 ans.

Tableau 1 : Genre des acteurs

\begin{tabular}{cccc}
\hline & Genre & Effectif & Pourcentage (\%) \\
\hline \multirow{2}{*}{ Eleveurs } & Homme & 47 & 94 \\
& Femme & 3 & 6 \\
\multirow{3}{*}{ Cultivateurs } & Homme & 62 & 69 \\
& Femme & 28 & 31 \\
\hline
\end{tabular}

Tableau 2: Age des acteurs

\begin{tabular}{|c|c|c|c|}
\hline & Genre & Effectif & Pourcentage (\%) \\
\hline \multirow{3}{*}{ Eleveurs } & 20 à 40 ans & 11 & 22 \\
\hline & 41 à 50 ans & 24 & 48 \\
\hline & Plus de 50 ans & 15 & 30 \\
\hline \multirow{3}{*}{ Cultivateurs } & 20 à 40 ans & 22 & 24 \\
\hline & 41 à 50 ans & 36 & 40 \\
\hline & Plus de 50 ans & 32 & 36 \\
\hline
\end{tabular}

\section{Nationalité et situation matrimoniale}

Les figures 2, 3, 4 et 5 présentent la nationalité et la situation matrimoniale des éleveurs et des cultivateurs. La majorité des cultivateurs sont des nationaux à $72 \%$ contre $28 \%$ de non nationaux et majoritairement mariés à $92 \%$ contre $8 \%$ de célibataires. Quant aux éleveurs, $76 \%$ sont des nationaux généralement sénoufo, $24 \%$ sont des non nationaux peulhs burkinabés et maliens. La quasi-totalité de ces éleveurs sont mariés à $98 \%$ contre $2 \%$ de célibataires. 


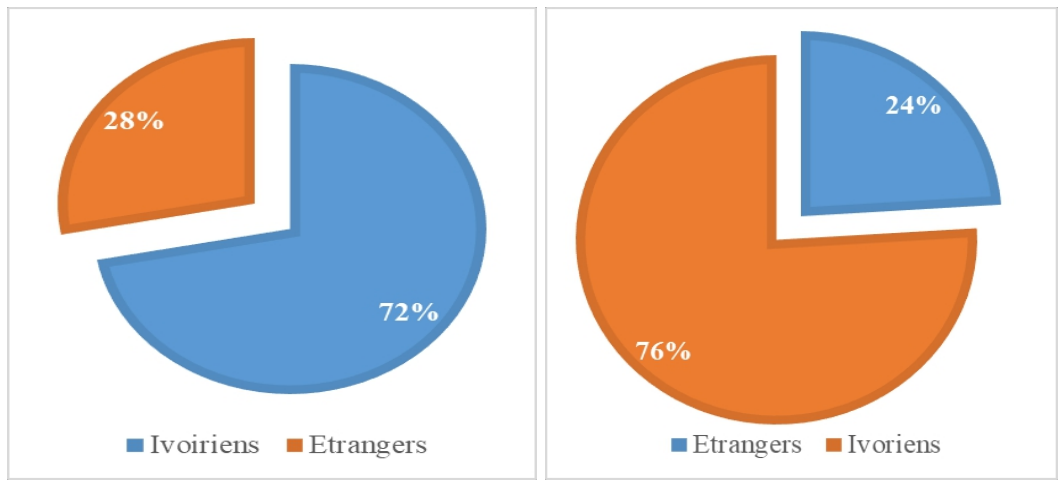

Figure 2 : Nationalité des cultivateurs

Figure 3 : Nationalité des éleveurs
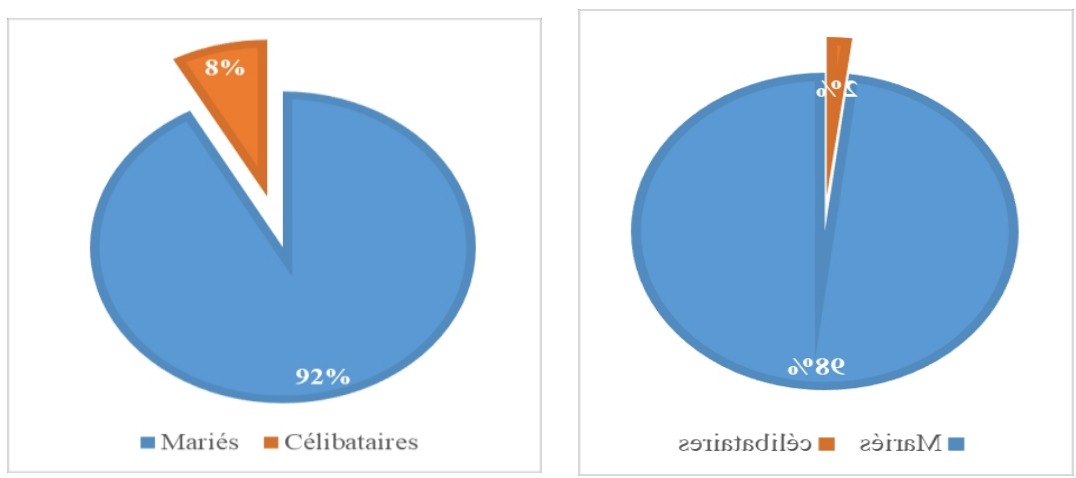

Figure 4: Situation matrimoniale

Figure 5: Situation matrimoniale des éleveurs des cultivateurs

\section{Activités des éleveurs}

\section{Caractéristiques des bovins}

Le tableau 3 indique les différentes caractéristiques des bovins rencontrés sur le terrain. La race bovine la plus représentée dans l'ensemble est constituée par les Métis (34,06\%). Viennent ensuite, les Zébus (28,46\%). La race bovine la moins représentée est constituée par les N'dama (16,48\%). Sur l'ensemble des troupeaux les femelles étaient majoritaires avec une proportion de $68,69 \%$. Les mâles ne représentaient que $31,31 \%$ avec une majorité de taurillons et la présence d'un mâle dominant. L'âge de ces bovins était tel que 16,86\% avaient moins de 1 an, 21,6\% avaient un âge compris entre 1 et 2 ans. Les plus âgés dont l'âge variait entre 2 et 3 ans puis ceux ayant plus de 3 ans représentaient respectivement $24,16 \%$ et $37,37 \%$.

Tableau 3 : Caractéristiques des bovins

\begin{tabular}{cll}
\hline Désignations & Effectif & Pourcentage (\%) \\
\hline Zébus & 183 & 28,46 \\
Race N'dama & 106 & 16,48 \\
Baoulé & 135 & 21 \\
Métis & 219 & 34,06
\end{tabular}




\begin{tabular}{llll}
\hline \multirow{2}{*}{ Sexe } & Mâle & 201 & 31,31 \\
& Femelle & 442 & 68,69 \\
\hline \multirow{4}{*}{ Age } & 0 à 1 an & 108 & 16,86 \\
& 1 à 2 ans & 139 & 21,6 \\
& 2 à 3 ans & 155 & 24,16 \\
& Plus de 3 ans & 241 & 37,37 \\
\hline
\end{tabular}

\section{Conduite et gestion de l'élevage}

Le tableau 4 présente la gestion et la conduite d'élevage pratiquées par les éleveurs. Les résultats ont montré que $42 \%$ des éleveurs de bovins dormaient dans des parcs de nuit privés près des habitations des propriétaires, pendant que 50\% de ces éleveurs avaient leurs bovins qui dormaient dans des parcs communautaires. Chez $8 \%$ d'éleveurs les parcs étaient inexistants. Les éleveurs nourrissaient à $100 \%$ leurs bovins de fourrage naturel généralement pendant la divagation. Par ailleurs, 35,3\% parmi eux apportaient un complément alimentaire en plus du fourrage naturel. Par principe, tous les éleveurs utilisent à $100 \%$ les cours d'eau et les marigots pour abreuver les bovins. En plus, $14 \%$ utilisaient les barrages et $22 \%$ avaient recours aux abreuvoirs à domicile pour renforcer l'abreuvement de leurs troupeaux. La majorité des bovins était soumise à un traitement vétérinaire à raison de $94 \%$ des éleveurs contre $6 \%$ qui ne recevaient aucun soin. L'hygiène dans laquelle vivaient les bovins était bonne chez 54\% des éleveurs et mauvaise chez $46 \%$ parmi eux.

Tableau 4 : Gestion des bovins

\begin{tabular}{cccc}
\hline \multicolumn{2}{c}{ Désignations } & Effectif & Pourcentage (\%) \\
\hline \multirow{3}{*}{ Habitat } & Parc de nuit (Privé) & 21 & 42 \\
& Parc communautaire & 25 & 50 \\
& Inexistant & 4 & 8 \\
\hline \multirow{2}{*}{ Mode d'alimentation } & Fourrage naturel & 50 & 100 \\
& Complémentation & 17 & 35,3 \\
\hline \multirow{2}{*}{ Abreuvement } & Cours d'eaux & 50 & 100 \\
& Barrages & 7 & 14 \\
& Abreuvoirs & 11 & 22 \\
\hline \multirow{2}{*}{ Suivi sanitaire } & Soins vétérinaires & 47 & 94 \\
& Inexistant & 3 & 6 \\
\hline \multirow{2}{*}{ Hygiène des animaux } & Bonne & 27 & 54 \\
& Mauvaise & 23 & 46 \\
\hline
\end{tabular}

\section{Conduite de la divagation des bovins}

Le tableau 5 présente l'organisation de la divagation des bovins. En période de saison sèche, la divagation des bovins débutait à 9 heures et prenait fin à 18 heures soit 9 heures de temps. La distance parcourue était de 7 à 8 kilomètres. Par contre, en période de saison des pluies où il $\mathrm{y}$ avait suffisamment d'herbes, les bovins parcouraient 3 à 4 kilomètres pour satisfaire leur ration journalière. Ainsi $58 \%$ des bovins étaient satisfaits après 5 heures 
de divagation (9h-14h) et 42\% consommaient l'herbe jusqu'à 17 heures soit 8 heures de divagation pour éviter toute dispute entre paysans et éleveurs.

Tableau 5 : Gestion de la divagation des bovins

\begin{tabular}{|c|c|c|c|c|c|}
\hline & $\begin{array}{c}\text { Distance } \\
\text { parcourue }\end{array}$ & $\begin{array}{c}\text { Heure de } \\
\text { divagation }\end{array}$ & Effectif & $\begin{array}{c}\text { Heure } \\
\text { d'abreuvement }\end{array}$ & $\begin{array}{c}\text { Pourcentage } \\
(\%)\end{array}$ \\
\hline Saison sèche & 7 à $8 \mathrm{~km}$ & $9 \mathrm{~h}$ à $18 \mathrm{~h}$ & 50 & $12 \mathrm{~h}$ à $14 \mathrm{~h}$ & 100 \\
\hline Saison des & 3 à 4 km & $9 \mathrm{~h}$ à $14 \mathrm{~h}$ & 29 & $12 \mathrm{~h}$ à $14 \mathrm{~h}$ & 58 \\
\hline pluies & 3 à 4 km & $9 \mathrm{~h}$ à $17 \mathrm{~h}$ & 21 & $12 \mathrm{~h}$ à $14 \mathrm{~h}$ & 42 \\
\hline
\end{tabular}

\section{Effectif des bovins en divagation}

Le tableau 6 montre les effectifs des bovins en divagation. Les éleveurs dont les effectifs de bovins étaient compris entre 31 et 40 têtes, étaient les plus nombreux (40\%). Viennent ensuite, ceux dont les effectifs sont au-delà de 40 têtes $(36 \%)$. Les éleveurs dont les effectifs de bovins sont en deçà de 10 têtes sont les moins nombreux.

Tableau 6 : Effectifs des bovins

\begin{tabular}{ccc}
\hline Nombre de têtes & Effectif & Pourcentage (\%) \\
\hline 1 à 10 & 5 & 10 \\
11 à 30 & 7 & 14 \\
31 à 40 & 20 & 40 \\
Plus de 40 & 18 & 36 \\
\hline
\end{tabular}

\section{Motifs de la divagation des bovins}

Le tableau 7 présente les raisons pour lesquelles les éleveurs soumettaient leurs bovins à la divagation. Tous les éleveurs (100\%) ont opté pour la divagation dans le but de trouver du pâturage pour nourrir leurs animaux et ensuite un point d'eau pour les abreuver. Cependant, 8\% de ces éleveurs dans certains villages n'avaient pas aménagé de parcs pour leurs bovins, d'où, la divagation à tout moment de la journée comme dans la nuit.

Tableau 7 : Motifs de divagation des bovins

\begin{tabular}{ccc}
\hline Motifs & Effectif & Pourcentage $(\%)$ \\
\hline Absence de parcs & 4 & 8 \\
Recherche de pâturage & 50 & 100 \\
Recherche de point d'eau & 50 & 100 \\
\hline
\end{tabular}

\section{Activités des cultivateurs}

Le tableau 8 présente les différentes superficies des cultures pratiquées par les cultivateurs. Pour les cultures pérennes et les cultures annuelles, les cultivateurs dont les superficies sont au-delà de deux hectares, sont les plus nombreux $(47 \%)$ pour les cultures pérennes et $43 \%$ pour les cultures annuelles. Viennent ensuite, ceux dont la superficie est comprise entre 1 et 2 hectares $(30 \%)$ pour les cultures pérennes et $37 \%$ pour les cultures annuelles. Quant aux cultures maraîchères, les superficies les plus exploitées sont les 
parcelles de moins de $500 \mathrm{~m}^{2}$, soit, $60 \%$ des cultivateurs. Viennent ensuite, les superficies comprises entre $500 \mathrm{~m}^{2}$ et 1 hectare, soit, $30 \%$ des cultivateurs.

Tableau 8 : Superficies des différentes cultures

\begin{tabular}{cccc}
\hline Cultures & Superficies & Effectifs & Pourcentage $(\%)$ \\
\hline \multirow{4}{*}{ Pérennes } & 0 à 1 ha & 7 & 23 \\
& 1 à 2 ha & 9 & 30 \\
& Plus de 2 ha & 14 & 47 \\
\hline \multirow{4}{*}{ Annuelles } & 0 à 1 ha & 6 & 20 \\
& 1 à 2 ha & 11 & 37 \\
& Plus de 2 ha & 13 & 43 \\
\hline \multirow{3}{*}{ Marâichères } & 0 à 500 m2 & 18 & 60 \\
& 501 à 1 ha & 9 & 30 \\
& Plu de 1 ha & 3 & 10 \\
\hline
\end{tabular}

\section{Types et parties des cultures endommagées par les bovins}

\section{Cultures pérennes}

Les figures 6 et 7 présentent l'incidence de la divagation des bovins sur les cultures pérennes. L'anacarde est la plus endommagée par la divagation des bovins $(87 \%)$ contre $70 \%$ pour les mangues. Les parties les plus endommagées sont les fruits $(80 \%)$. Les tiges et les feuilles sont les moins endommagées avec $4 \%$ pour chacune.

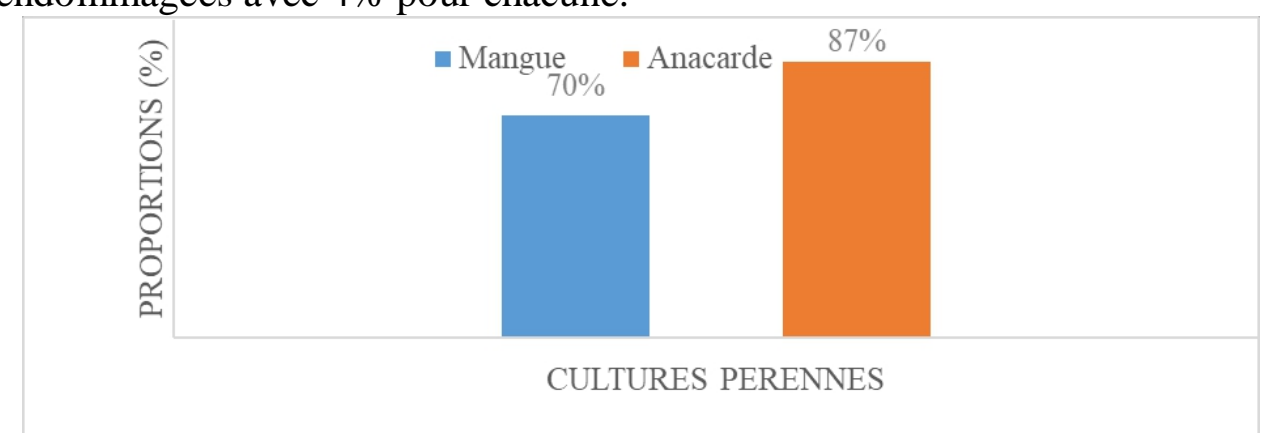

Figure 6 : Evaluation des dégâts sur les différentes cultures pérennes
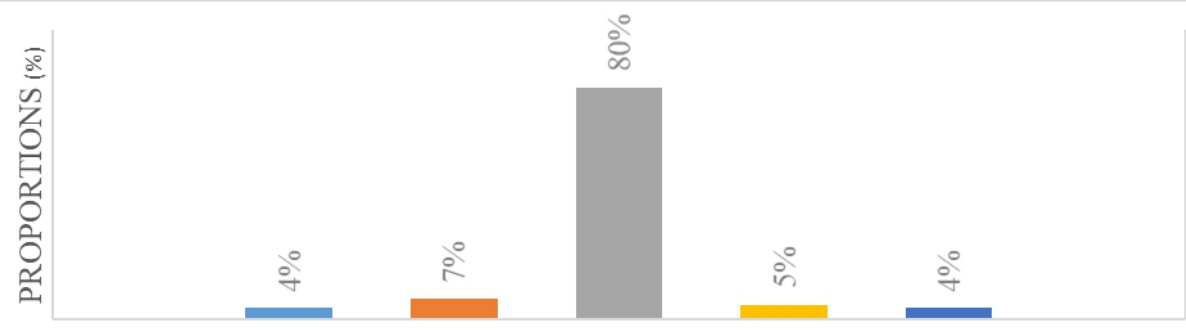

PARTIES DES CULTURES PÉRENNES

घeuilles $\square$ Fleurs $\square$ Fruits $\square$ Racines $\square$ Tiges

Figure 7 : Parties des cultures pérennes endommagées par les bovins 


\section{Cultures annuelles}

Les figures 8 et 9 indiquent l'incidence de la divagation des bovins sur les cultures annuelles. Le coton est le plus endommagée par la divagation des bovins $(73 \%)$ contre $65 \%$ pour le maïs, $60 \%$ pour de riz et $57 \%$ pour l'arachide. Les parties les plus endommagées sont les fruits (35\%), les feuilles $(27 \%)$ et les tiges (25\%). Les fleurs (10\%) et les racines (3\%) sont les moins endommagées.

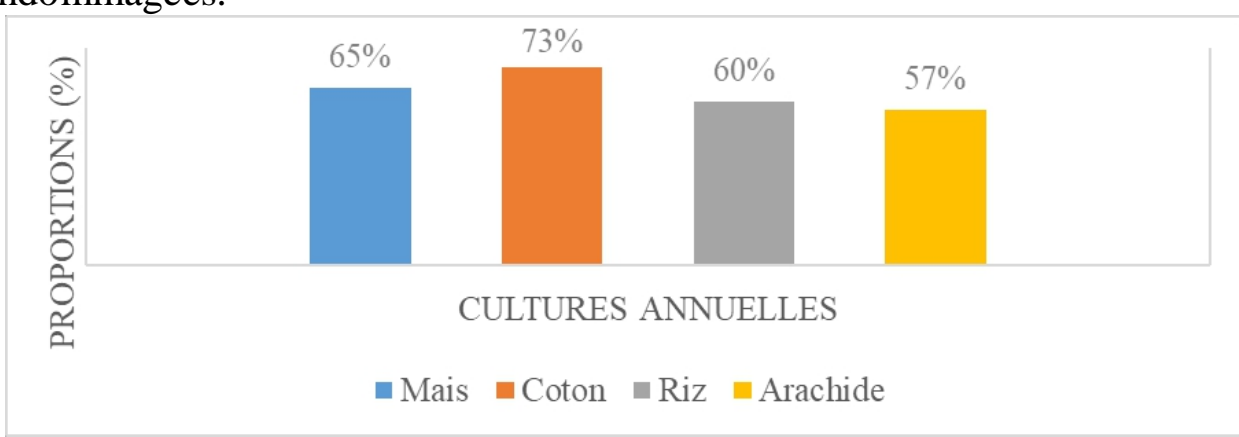

Figure 8 : Evaluation des dégâts sur les différentes cultures annuelles

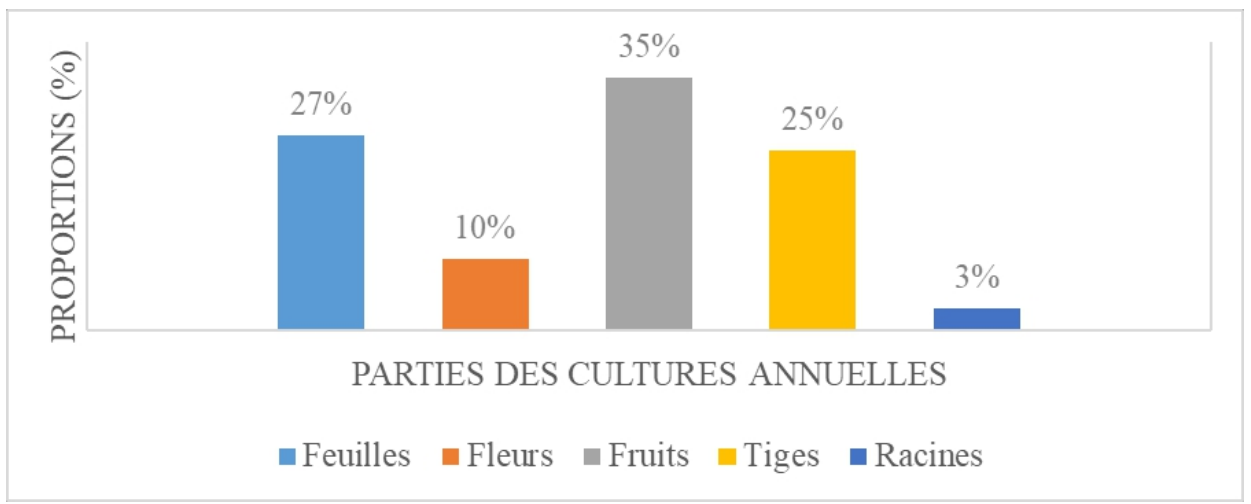

Figure 9 : Parties des cultures annuelles endommagées par les bovins

\section{Cultures maraîchères}

Les figures 10 et 11 montrent l'impact de la divagation des bovins sur les cultures maraîchères. Le gombo (76\%) et la tomate $(60 \%)$ ont été les plus endommagées par la divagation des bovins contre 55\% pour la laitue, 33\% pour le chou, $26 \%$ pour la carotte et $20 \%$ pour le concombre. Les parties les plus endommagées sont les fruits (70\%). Les feuilles (20\%) et les fleurs (10\%) sont les moins endommagées. 


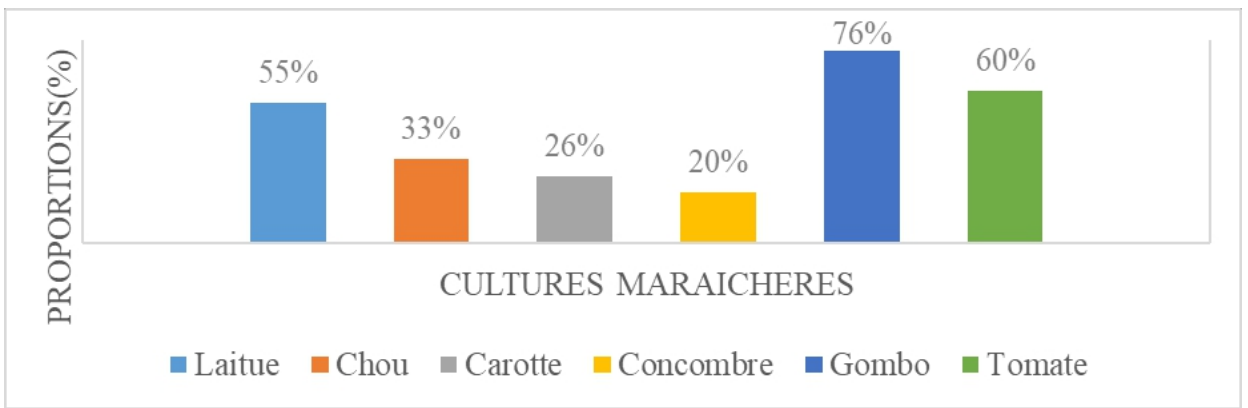

Figure 10 : Evaluation des dégâts sur les différentes cultures maraîchères

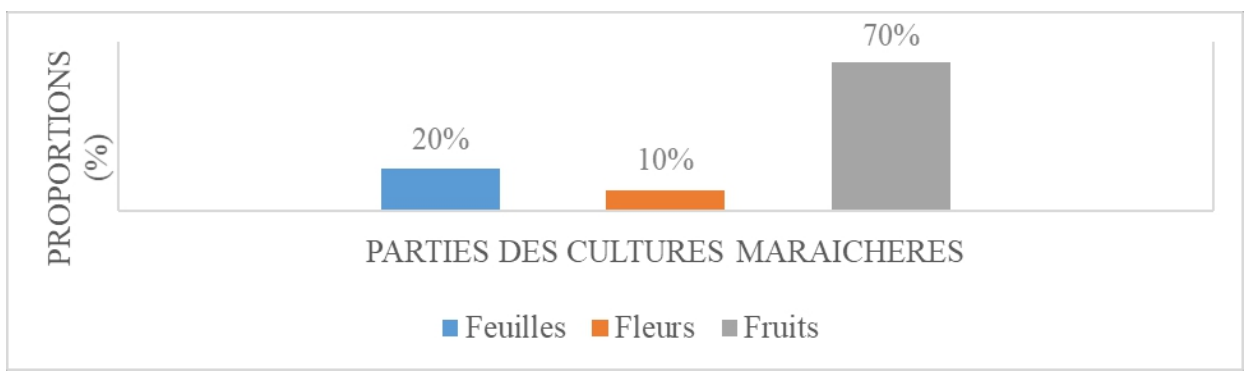

Figure 11 : Parties des cultures maraîchères endommagées par les bovins

\section{Importance des dégâts des cultures}

La figure 12 présente l'importance des dégâts sur les différentes cultures. Les cultures annuelles ont été les plus endommagées par les bovins avec un taux de $45 \%$ des dégâts enregistrés, viennent ensuite les cultures maraîchères avec un taux de $30 \%$ et enfin les cultures pérennes avec une proportion de $25 \%$.

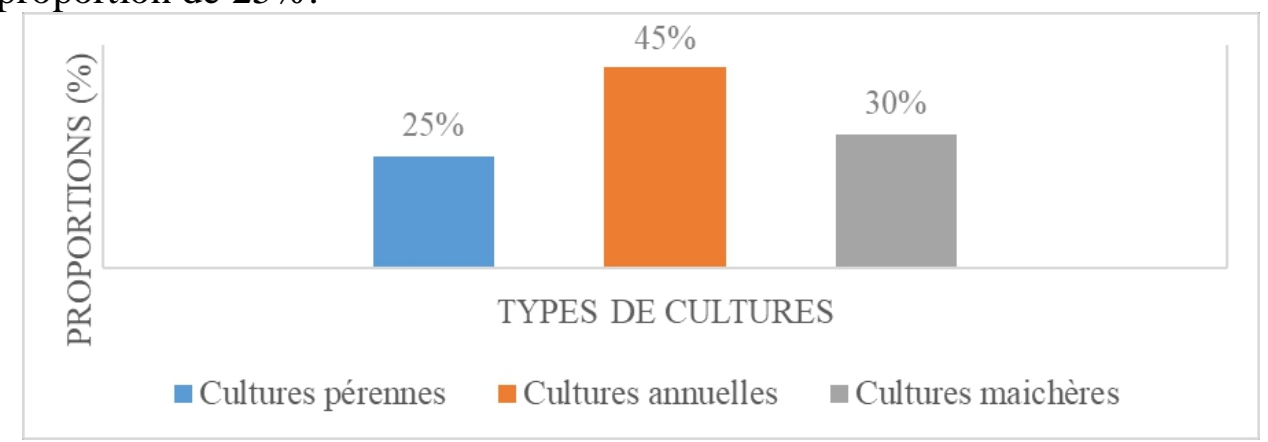

Figure 12 : Importance des dégâts selon le type de culture

\section{Causes des dégâts des cultures}

\section{Causes liées aux éleveurs}

La figure 13 présente les raisons liées aux éleveurs. La majorité des dégâts liés aux éleveurs soit $35 \%$ étaient dus à une insuffisance du nombre d'éleveurs pour suivre les bovins, $29 \%$ pour la méconnaissance des terroirs, 
$21 \%$ pour la méconnaissance du calendrier cultural de localités et $15 \%$ pour un manque de couloir de passage des bovins.

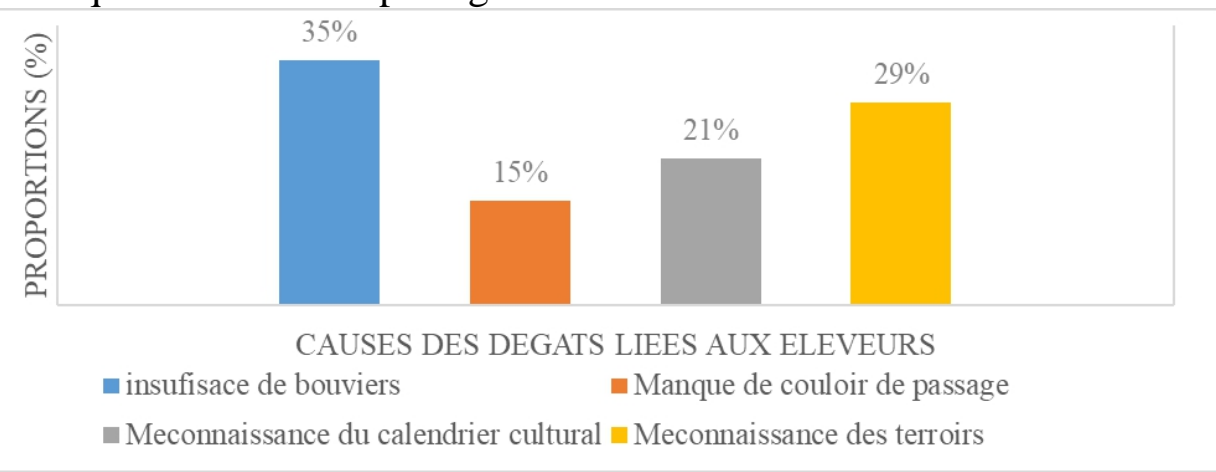

Figure 13 : Causes des dégâts liées aux éleveurs

\section{Causes liées aux cultivateurs}

La figure 14 présente les différentes causes des dégâts liées aux cultivateurs. La responsabilité des cultivateurs dans les dégâts causés par les bovins est telle que $40 \%$ sont dus à une absence de clôtures vives des parcelles cultivées, $33 \%$ au stockage des récoltes sur les champs et $27 \%$ à l'absence de limitation des terres.

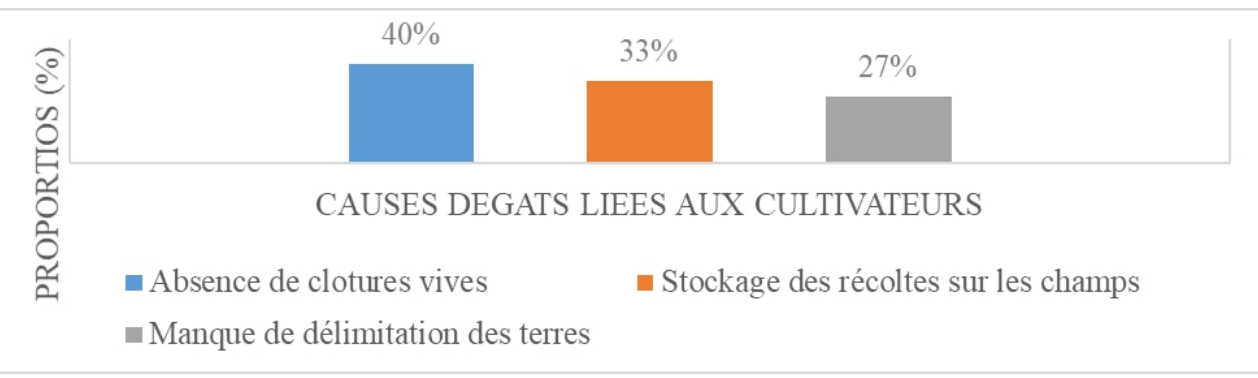

Figure 14 : Causes des dégâts liées aux cultivateurs

\section{Autres causes}

La figure 15 indique d'autres raisons des dégâts causés par les bovins. La cause essentielle a été l'absence ou l'insuffisance des pâturages (55\%) contre $45 \%$ à l'éloignement des points d'abreuvement.

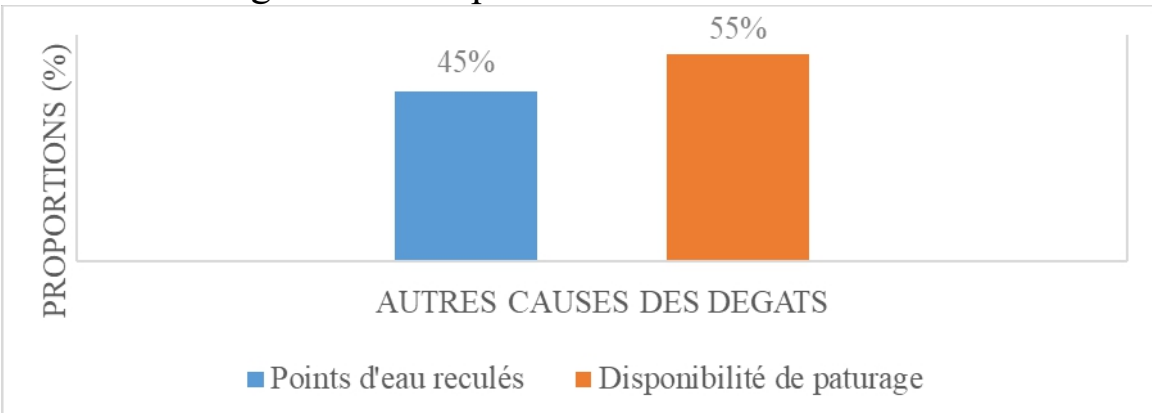

Figure 15 : Autres causes des dégâts des cultures 


\section{Conséquences de la divagation des bovins}

\section{Conséquences sur les bovins}

La figure 16 montre les conséquences de la divagation des bovins sur les bovins eux-mêmes. Ces conséquences étaient des empoisonnements, des abattages, des contaminations à travers les herbes consommées et les misesbas en pleine brousse dans des proportions relatives de $32 \%, 28 \%$, 15\%, et $25 \%$.

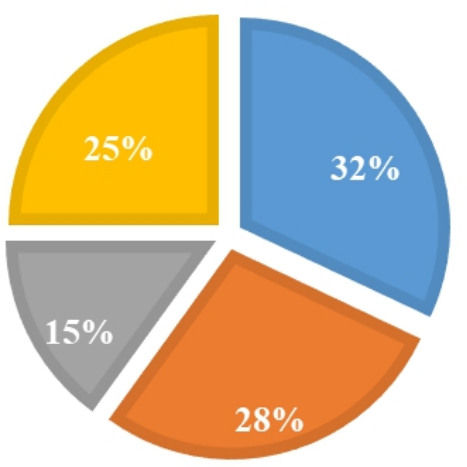

Empoisonnement $\quad$ Abattage des bovins $\quad$ contamination $=$ Mise bas

Figure 16 : Répartitions des conséquences sur les bovins

\section{Conséquences sur les cultures}

Le tableau 9 présente les superficies des différentes cultures détruites par les bovins. Une superficie totale de 8,1 ha de cultures a été détruite dont $31 \%$ de cultures pérennes, $59 \%$ de cultures annuelles et $10 \%$ de cultures maraichères.

Tableau 9 : Superficies des cultures détruites

\begin{tabular}{ccccc}
\hline & Cultures pérennes & Cultures annuelles & Cultures maraîchères & Total \\
\hline Superficies & 2,5 ha & 4,8 ha & $800 \mathrm{~m}^{2}$ & 8,1 ha \\
Pourcentage $(\%)$ & 31 & 59 & 10 & 100 \\
\hline
\end{tabular}

\section{Avantages de la divagation des bovins}

La figure 17 montre les avantages de la divagation des bovins. La divagation a permis dans $66 \%$ des cas aux éleveurs de faire des économies sur l'achat des aliments et $34 \%$ des avantages étaient des possibilités de fertilisation des champs et des espaces de pacage des bovins. 


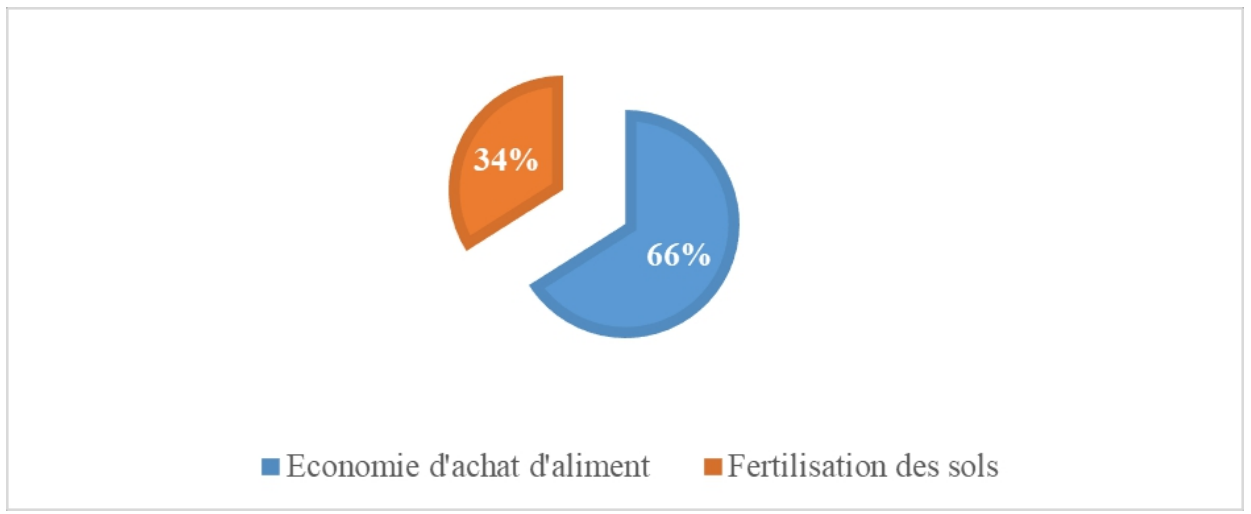

Figure 17 : Avantages de la divagation des bovins

\section{Discussion}

La majorité des acteurs (éleveurs et cultivateurs) sont des hommes (94\% des éleveurs et $69 \%$ des cultivateurs) contre $6 \%$ et $31 \%$ respectivement pour les éleveurs et les cultivateurs qui sont des femmes. Ce résultat est contraire à celui d'Akaffou (2013) qui stipule que les femmes mariées, en plus de leur fonction domestique participent à la production agricole familiale. Les éleveurs et cultivateurs sont en majorité des ivoiriens contre une minorité d'étrangers. La faible intervention des non nationaux dans l'agriculture est due à un accès difficile aux parcelles cultivables. Ce résultat est similaire à celui de Ouattara (2009) qui stipule que les exploitations appartiennent pour la plupart à des ivoiriens agriculteurs, fonctionnaires ou commerçants qui investissent dans ce mode d'élevage. Les métis constituent la race majoritaire de bovins élevés. Cela pourrait s'expliquer par la trypanotolérance de cette race qui s'adapte mieux aux conditions climatiques du Nord de la Côte d'Ivoire et particulièrement de Korhogo. Les femelles sont dominantes dans les troupeaux. Cette situation serait due au sexe ratio, qui est d'un mâle pour 20 à 25 femelles. De plus, les mâles sont engraissés pour la boucherie alors que les femelles sont conservées pour la reproduction. Chabi (2016) confirme ce résultat en disant que la structure du sexe des troupeaux bovins montre une forte proportion des femelles soit $76 \%$ dont $47 \%$ de vaches. La plupart des bovins sont âgés de plus de 3 ans. La présence massive des bovins âgés serait liée à la culture attelée. Kohlhagen (2002), ne partage pas cet avis lorsqu'il dit que l'âge des bovins de trait est de 2 à 3 ans. Plus de la moitié des bovins passent la nuit dans des parcs communautaires. Cette situation serait due au fait que ce sont les éleveurs qui ont généralement la garde des bovins de plusieurs propriétaires. Tous les éleveurs nourrissent leurs bovins de fourrage naturel et utilisent les cours d'eau disponibles pour abreuver les animaux. Cette situation pourrait se justifier par le souci des éleveurs de faire des économies sur l'achat des compléments alimentaires. L'utilisation importante des cours d'eau et marigots est probablement due à l'éloignement des barrages 
dans la zone pour l'abreuvement des bovins. Les éleveurs ont recours aux soins vétérinaires dans le suivi médical des bovins. La proportion considérable des soins vétérinaires s'expliquerait par la présence des pathologies et des tiques qui sévissent dans la zone. Bonfoh (2003) a montré qu'au niveau des doses d'antibiotiques, les éleveurs injectent de faibles doses dans la lutte contre les maladies transmises par les tiques.

En période de sècheresse, la divagation des bovins dure $9 \mathrm{~h}$ sur 7 à 8 $\mathrm{km}$ contre $4 \mathrm{~h}$ en saison pluvieuse sur 3 à $4 \mathrm{~km}$. La durée de la divagation des bovins serait donc liée à la disponibilité de l'herbe fraîche. Ce résultat concorde avec celui de Dehoux et d'Hounsou (2011), qui stipulent qu'en saison sèche, les animaux se déplacent après la traite matinale vers 8 à 9 heures et reviennent vers 18 à 19 heures, soit à peu près 10 heures de pâturage. Les superficies allouées aux cultures annuelles et pérennes sont plus importantes que celles des cultures maraîchères. Cela pourrait s'expliquer par le fait que les cultures maraichères sont le plus souvent exercées par les femmes dans les bas-fonds. Ce résultat est en accord avec celui de Michel (2015), qui indique que toutes les terres sont mises en valeur et rares sont les espaces vides, à l'exception des bois sacrés. Toutes les cultures subissent des dommages de la part des bovins en divagation. Les parties les plus endommagées sont les fruits et des feuilles quel que soit le type de culture. L'importance des dégâts observés au niveau des différentes catégories de cultures chez les cultivateurs est due au manque de haies vives pour assurer la protection des cultures. Ce résultat est contraire à celui de Véronique (1997) qui affirme que les jardins maraîchers qui apparaissent un peu partout sont généralement protégés par des palissades en bois et branchages contre la divagation des animaux. Les causes des dégâts des cultures résultant de la divagation des bovins sont multiples et liées aussi bien aux éleveurs qu'aux cultivateurs. La principale cause des dégâts est essentiellement due à la négligence des différents acteurs. L'étendue des parcelles cultivées ainsi que l'abondance des récoltes compliqueraient la tâche des cultivateurs confrontés à une main d'œuvre insuffisante. Ce résultat est contraire à celui de Kohlhagen (2002) qui stipule que traditionnellement, ces cultures sont protégées des animaux par une clôture faite de branches d'arbres épineux. Les conséquences de la divagation des bovins s'observent sur les bovins eux-mêmes ainsi que sur les cultures. Ces conséquences sont probablement dues au refus de la responsabilité des dégâts causés par les bovins au niveau des éleveurs (Dehoux et Hounsou, 2011). L'importance des dommages observés au niveau des cultures annuelles s'expliquerait par le fait qu'elles sont les plus exercées dans ladite commune.

\section{Conclusion}

Au terme de notre étude, il convient de retenir que les bovins causent d'énormes dégâts sur les cultures annuelles, pérennes et maraîchères. Les 
cultures annuelles sont les plus attaquées et les dégâts se situent aussi bien au niveau des fleurs, des fruits et des feuilles. La divagation des bovins entrainent aussi des dégâts à leur niveau dont les empoisonnements et les abattages. Cette situation entraine d'énormes pertes économiques aussi bien chez les cultivateurs que chez les éleveurs qui endossent la responsabilité. Vivement que tous les partenaires du monde agricole collaborent afin de trouver des solutions idoines car il y va de l'intérêt de tous. Eleveurs et agriculteurs pourraient augmenter leurs revenus et les populations seraient à l'abri de la pénurie ou la cherté des denrées alimentaires d'origine animale et végétale. Les autorités locales contribueraient à la gestion de la divagation des animaux par la création de couloirs de transhumance et de barrages pour les points d'eau. Des études complémentaires pourraient être menées afin de chiffrer les pertes économiques liées à la divagation des bovins sur les différentes cultures dans le département de Korhogo.

\section{Remerciements}

Les remerciements vont à l'endroit des zootechniciens de l'Institut de Gestion Agropastorale de l'Université Peleforo GON COULIBALY de Korhogo pour la collaboration dans la collecte des données. Nous remercions également les propriétaires des élevages bovins et leurs bouviers ainsi que tous les cultivateurs installés dans la ville de Korhogo qui nous ont permis d'avoir accès aux animaux et aux différentes cultures.

\section{Conflit D'interet}

Les auteurs ne déclarent aucun conflit d'intérêt.

\section{References:}

1. Akaffou N. A., 2013. Elevage bovin laitier dans la région du Poro (nord Côte d'Ivoire) : situation actuelle et perspective $\mathrm{s}$ de développements. Thèse du doctorat en médecine vétérinaire, université cheikh Anta Diop de Dakar, 113p.

2. Anonyme, 2014. Recensement Général de la Population et de l'Habitat ; Direction régionale du district des savanes. Institut Nationale de la Statistique, 35p.

3. Bonfoh B., 2003. Les sources de contamination du lait local et les méthodes d'amélioration de sa qualité microbiologique à Bamako (mali). Lait sain pour le sahel, 33-34p.

4. Chabi L., 2016. Demography and zootechnical performance of traditional cattle farming in Northern Benin [in French]. Rev. Elev. Med. Vet. Pays Trop., 69p.

5. Couibaly D., 2013. Politique de développement de l'élevage en Côte d'Ivoire : direction de la planification des programmes (DPP), 4p. 
6. Dehoux J. P. et Hounsou G., 2011. Projet de développement pastoral intégré dans le Borgou, phase II, 77p.

7. Guen T., 2008. Le développement agricole et pastoral du Nord de la Côte-d'Ivoire : problèmes de coexistence, Les Cahiers d'Outre-Mer, $18 \mathrm{p}$.

8. Kohlhagen D., 2002. Gestion foncière et conflits entre agriculteurs et éleveurs, autochtones et étranger dans la région de Korhogo (Côte d'Ivoire). Rapport de mission dans le cadre du programme de recherche vers de nouvelle dynamique entre loi et coutume, CIRAD, 2002, 33p.

9. Lhoste P. et Milleville P., 2004. La conduite des animaux : Techniques et pratiques des éleveurs, 231-251p.

10. Michel B., 2015. Elevage et problème de divagation des bêtes en milieu urbain, $11 \mathrm{p}$.

11. Ouattara C., 2009. Contribution à l'amélioration de l'élevage sédentaire bovin dans la région de Korhogo. Mémoire DUT. Côte d'Ivoire, 26p.

12. Véronique A., 1997. Les peuls transhumants du Nord de la Côte d'Ivoire entre l'Etat et les paysans : la mobilité en réponse aux crises, 670-687p.

13. Yeo N., 2017. Occurrence of Babesia spp in bovine breeding in Poro area (Côte d'Ivoire). International Journal of Agronomy and Agricultural Research (IJAAR) 8p. 